\title{
Digestive enzymes of fungal origin as a relevant cause of false positive Aspergillus antigen testing in intensive care unit patients
}

\author{
Ines Schroeder ${ }^{1} \cdot$ Karl Dichtl $^{2} \cdot$ Uwe Liebchen $^{1} \cdot$ Johannes Wagener $^{3,4} \cdot$ Michael Irlbeck $^{1} \cdot$ Michael Zoller $^{1}$. \\ Christina Scharf ${ }^{1}$
}

Received: 3 July 2020 / Accepted: 12 August 2020 / Published online: 3 September 2020

(c) The Author(s) 2020

\begin{abstract}
Background Galactomannan antigen (GM) testing is widely used in the diagnosis of invasive aspergillosis (IA). Digestive enzymes play an important role in enzyme substitution therapy in exocrine pancreatic insufficiency. As digestive enzymes of fungal origin like Nortase contain enzymes from Aspergillus, a false-positive result of the test might be possible because of cross-reacting antigens of the cell wall of the producing fungi. We, therefore, asked whether the administration of fungal enzymes is a relevant cause of false-positive GM antigen test results.

Methods Patients with a positive GM antigen test between January 2016 and April 2020 were included in the evaluation and divided into two groups: group 1-Nortase-therapy, group 2-no Nortase-therapy. In addition, dissolved Nortase samples were analyzed in vitro for GM and $\beta-1,3-\mathrm{D}$-glucan. For statistical analysis, the chi-squared and Mann-Whitney $U$ tests were used.

Results Sixty-five patients were included in this evaluation (30 patients receiving Nortase and 35 patients not receiving Nortase). The overall false positivity rate of GM testing was $43.1 \%$. Notably, false-positive results were detected significantly more often in the Nortase group (73.3\%) than in the control group $(17.1 \%, p<0.001)$. While the positive predictive value of GM testing was 0.83 in the control group, there was a dramatic decline to 0.27 in the Nortase group. In vitro analysis proved that the Nortase enzyme preparation was highly positive for the fungal antigens GM and $\beta-1,3$-D-glucan.

Conclusions Our data demonstrate that the administration of digestive enzymes of fungal origin like Nortase leads to a significantly higher rate of false-positive GM test results compared to that in patients without digestive enzyme treatment.
\end{abstract}

Keywords Invasive aspergillosis · Critical illness · Galactomannan antigen assay $\cdot$ False positive results · Nortase · Digestive enzymes of fungal origin

Ines Schroeder and Karl Dichtl contributed equally to this project and are, therefore, both considered first authors.

Ines Schroeder

Ines.Schroeder@med.uni-muenchen.de

1 Department of Anesthesiology, University Hospital, LMU München, Marchioninistrasse 15, 81377 Munich, Germany

2 Max Von Pettenkofer-Institut für Hygiene Und Medizinische Mikrobiologie, LMU München, Medizinische Fakultät, Munich, Germany

3 Institut für Hygiene Und Mikrobiologie, Julius-Maximilians-Universität Würzburg, Würzburg, Germany

4 National Reference Center for Invasive Fungal Infections (NRZMyk), Jena, Germany

$\begin{array}{ll}\text { Abbreviations } \\ \text { BAL } & \text { Bronchoalveolar lavage } \\ \text { BDG } & (1,3) \text {-Beta-D-glucan } \\ \text { CT } & \text { Computed tomography } \\ \text { DEFO } & \text { Digestive enzymes of fungal origin } \\ \text { ELISA } & \text { Enzyme-linked immunosorbent assay } \\ \text { GM } & \text { Galactomannan } \\ \text { IA } & \text { Invasive aspergillosis } \\ \text { ICU } & \text { Intensive care unit } \\ \text { PPV } & \text { Positive predictive value }\end{array}$

\section{Introduction}

Invasive aspergillosis (IA) is a significant cause of morbidity and mortality in immunocompromised patients [1]. Diagnosis of IA remains challenging. In 2019, the consensus 
committee of the European Organization for Treatment of Cancer/Mycoses and the Mycoses Study Group (EORTC/ MSG) proposed their revised definition for the diagnosis of invasive fungal infections. IA is diagnosed based on findings in imaging studies, host factors, histopathology, and microbiology [2]. Since the sensitivity of Aspergillus culture or microscopy in blood and bronchoalveolar lavage (BAL) is often poor, Aspergillus antigen testing is additionally recommended for patients who are at risk or suspicion of IA [3-5].

Galactomannan $(\mathrm{GM})$ is a polysaccharide and a major constituent of cell walls of Aspergillus species. It can be detected in the host's bloodstream during invasive infection [6]. The GM test is a double-sandwich enzyme-linked immunosorbent assay (ELISA), which is widely used for testing serum and BAL fluid for IA. Sensitivity of GM testing for IA ranges from 70 to $85 \%$, and specificity from 78 to $91 \%$, in immunocompromised patients [7]. Insufficient data are available regarding the test's performance in patients following solid organ transplantation. Sensitivity and specificity might be decreased in this setting, presumably due to less angioinvasive growth in patients with better immune defense compared to neutropenic patients $[8,9]$.

Digestive enzyme preparations are used for enzyme substitution therapy in exocrine pancreatic insufficiency [10]. The advantage of Nortase, a digestive enzyme of fungal origin (DEFO) in the ICU setting, in contrast to alternative formulas is that the capsule can be opened, and the contained powder is stable to gastric acidity. Therefore, enteral application through a feeding tube is feasible. Hence, it is the preferred substance used in enteral nutrition of patients with exocrine pancreas insufficiency, who are unable to take medication orally_in our setting, critically ill patients following major surgery. The powder contains fungal enzymes synthesized by Aspergillus oryzae that contains also GM in their cell wall like Aspergillus fumigatus. Enzymes derived from microbial sources can be obtained over the counter and have a long history of safe use within the food industry.

A high number of GM antigenemia episodes was noticed in patients receiving Nortase on the Intensive Care Units (ICU) of a University hospital. False positive results have not been associated with the administration of digestive enzymes before. The aim of this study was to investigate whether patients receiving Nortase have a higher rate of false-positive test results in GM testing.

\section{Methods}

\section{Study setting}

This is a monocentric, retrospective observational study investigating the influence of the application of Nortase on the performance of the GM ELISA. A local institutional review board approved the study (registration number 20-269).

\section{Laboratory testing}

GM measurement was performed using the Platelia Aspergillus antigen ELISA (Bio-Rad Laboratories, Hercules, CA, USA). Optical density (OD) indices were rounded to one decimal place, with a lower limit of 0.1. A GM OD index $\geq 0.5$ in two sequential samples including the retesting of the first positive sample was considered positive. $\beta-1,3-$ D-glucan (BDG) analysis was conducted using the Wako BDG assay (FUJIFILM Wako Chemicals Europe, Neuss, Germany).

We typically use Nortase (Repha, Langenhagen) as a digestive enzyme preparation in the clinical setting. Detailed product information, especially regarding the composition of the capsule, can be found in the summary of product characteristics.

In vitro analysis was performed on five Nortase samples. Nortase capsules were opened and suspended in $10 \mathrm{~mL}$ of normal saline each. The solution was agitated and subsequently processed like the serum samples. Three different batches were used to exclude contamination by batch.

\section{Study population}

All GM-seropositive patients treated at two ICUs of the university hospital of Munich between January 2016 and April 2020 were included in the evaluation. Subsequently, they were divided into two groups: group 1, receiving Nortase; and group 2, not receiving Nortase. Five to 15 capsules Nortase were administered two to four times daily determined by the attending physician. GM testing was performed twice a week, within the clinical routine of our ICUs. In addition, all patients receiving Nortase between January 2016 and April 2020 during their stay at the ICUs were evaluated for GM antigenemia.

\section{Data collection}

The charts of all patients were reviewed for the diagnosis of IA according to the criteria of the EORTC/MSG consensus group [2]. However, GM testing is a major factor in these criteria. In consideration of the question addressed by this study, the more restrictive categories "probable* IA" and "possible* IA" had to be applied, which are characterized by excluding GM from the criteria for categorization (Table 1). To strictly identify those patients who had no evidence of IA other than GM antigenemia with respect to the clinical characteristics of the study collective (ICU patients rather 
than hematology-oncology patients), an additional category was applied, i.e., "presumable IA". All patients who did not fulfill the EORTC/MSG criteria for IA but showed evidence for IA in two EORTC criteria (clinical features, mycological evidence other than GM, host factors) were declared as presumable. Accordingly, GM false positivity was defined as positive GM test results in patients without any further evidence of IA.

\section{Statistical analysis}

Statistical analysis was performed with IBM SPSS statistics (version 26.0, IBM Corp., Armonk, NY, USA). The dataset was evaluated with a focus on significant changes or differences in the rate of false-positive test results in both the groups. Therefore, the chi-squared test and the Mann-Whitney $U$ test were used.

\section{Results}

\section{GM antigenemia in DEFO patients}

Between January 2016 and April 2020, 40 patients at our ICUs received Nortase. Thirty (75\%) of them developed GM antigenemia and were included in the study.

\section{Demographic and clinical data of GM positive individuals}

In the study period, a total of 65 patients at our ICUs tested positive for GM. Thirty patients receiving Nortase were allocated to group 1, and 35 others were placed in group 2. As all patients were critically ill, immunocompromised, and often had undergone recent surgery, intense and invasive diagnostic procedures (for example, lung biopsies) were performed to rule out IA. For detailed patient characteristics and diagnostic workup, see Table 2.
The most frequent reason for the application of Nortase was the necessity of enteral feeding through an enteral feeding tube of patients with exocrine pancreatic insufficiency due to underlying cystic fibrosis following lung transplantation (27/30 patients). Three patients had different reasons for exocrine pancreatic insufficiency.

\section{Assessment of validity of GM testing}

In the Nortase group, patients were diagnosed with proven, probable*, possible* and presumable IA according to the (modified) EORTC/MSG criteria in 0, 4, 0 and 4 cases respectively (Fig. 1) [2]. Twenty-two cases positive for GM did not meet any of the clinical, histopathological or microbiological criteria, apart from antigenemia, allowing the diagnosis of proven, probable*, possible* or presumable IA. All of these cases underwent an intensive diagnostic workup, including CT scan and histopathology of the suspicious body site. Autopsies were performed on two deceased patients and showed no sign of IA. Consequently, these 22 patients (73.3\%) with GM antigenemia as the only parameter indicating IA were considered as false-positive.

In contrast, 8, 11, 1 and 9 patients allocated to group 2 were diagnosed with proven, probable*, possible*, and presumable IA, respectively, according to the (modified) EORTC/MSG criteria [2]. The remaining six patients $(17.1 \%)$ with GM antigenemia as the only parameter indicating IA were considered false positives (see Fig. 1). For details on patient characteristics classified as presumable IA, see Table 3 .

Including all patients, the positive predictive value of the GM antigen test was 0.57 . When comparing the two subgroups, the test performance in group 2 with a positive predictive value (PPV) of 0.83 was significantly better than in group 1 with a positive predictive value of $0.27(p<0.001)$.

Chi-squared analysis demonstrated that patients allocated to group 1 had a false-positive GM test significantly more often compared to group $2(p<0.001)$.
Table 1 EORTC/MSG and modified definitions of probability of invasive aspergillosis

\begin{tabular}{llll}
\hline Category & Host factor & Clinical feature & $\begin{array}{l}\text { Myco- } \\
\text { logical } \\
\text { evidence }\end{array}$ \\
\hline Probable IA & + & + & + \\
Probable* IA & + & + & $+*$ \\
Possible IA & + & + & - \\
Possible* IA & + & + & $-*$ \\
Presumable IA\# & \pm & \pm & $+*-$ \\
Proven IA & & Microscopic or cultural finding of Aspergillus spp. \\
& & from sterile material & \\
No evidence for IA & & $\leq 1$ criterion of probable IA* & \\
\hline
\end{tabular}

Note: Modified categories in italics; IA invasive aspergillosis; +* evidence other than GM seropositivity;

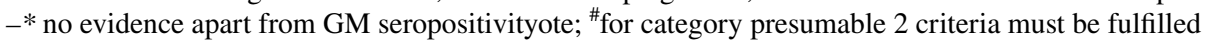


Table 2 Patient characteristics and diagnostic work up in both groups

Fig. 1 Distribution of falsepositive GM test results in both groups

\begin{tabular}{lll}
\hline & $\begin{array}{l}\text { Group1 "Nortase } \\
\text { therapy": } n(\%) \text { or } \\
\text { median [range] }\end{array}$ & $\begin{array}{l}\text { Group2 "no Nortase } \\
\text { therapy": } n \text { (\%) or } \\
\text { median [range] }\end{array}$ \\
\hline Number of patients & $30(46.2 \%)$ & $35(53.8 \%)$ \\
Age: years & $37[21,67]$ & $58[22,79]$ \\
Gender: male/female & $13 / 17(43.3 \% / 56.7 \%)$ & $19 / 16(54.3 \% / 45.7 \%)$ \\
Cystic fibrosis & $27(90 \%)$ & $2(5.7 \%)$ \\
Solid organ transplantation & $28(93.3 \%)$ & $19(54.3 \%)$ \\
Sepsis & $0(0 \%)$ & $7(17.1 \%)$ \\
ARDS & $0(0 \%)$ & $6(11.4 \%)$ \\
Microbiological workup (i.e., culture from BAL or sputum, & $30(100 \%)$ & $35(100 \%)$ \\
$\quad$ urine, blood, sterile body fluid samples) & $8(26.7 \%)$ & $26(74.3 \%)$ \\
Cultivation of Aspergillus spp. & $29(96.7 \%)$ & $35(100 \%)$ \\
CT of body site suspicious for IA & $4(13.3 \%)$ & $17(48.6 \%)$ \\
CT positive for IA* & $28(93.3 \%)$ & $24(68.6 \%)$ \\
Histopathology of biopsy from body site suspicious for IA & $10(28.6 \%)$ \\
Histopathology positive for invasive mold infection & $0(0 \%)$ & $35(100 \%)$ \\
Performed clinical examination & $30(100 \%)$ & $21(60 \%)$ \\
In-hospital mortality & $17(56.7 \%)$ & $3(8.6 \%)$ \\
Autopsy performed & $2(7 \%)$ & $2(5.7 \%)$ \\
Autopsy positive for IA & $0(0 \%)$ & $31(88.6 \%)$ \\
Antifungal therapy of IA & $24(80 \%)$ &
\end{tabular}

Note: $B A L$ bronchoalveolar lavage, $I A$ invasive aspergillosis, ARDS Acute respiratory distress syndrome, $C T$ Computed tomography

*According to the criteria of the EORTC/MSG consensus guidelines [2]

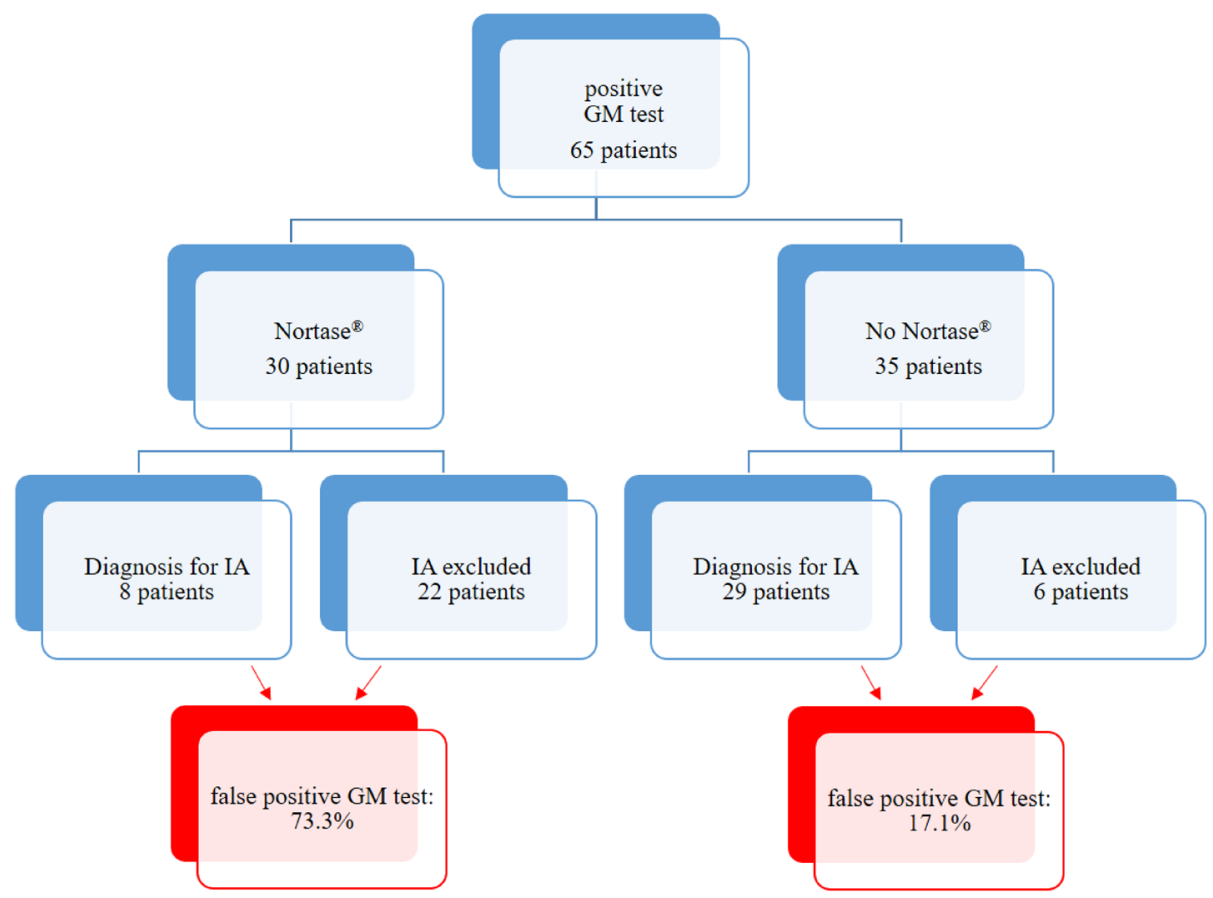

Note: IA, invasive aspergillosis; GM, galactomannan 
Table 3 Characteristics of patients classified as presumable IA

\begin{tabular}{|l|l|l|l|}
\hline $\begin{array}{l}\text { Patient (no.) } \\
\text { 1-4: Nortase } \\
5-13: \text { no Nortase }\end{array}$ & $\begin{array}{l}\text { Host } \\
\text { factors }\end{array}$ & $\begin{array}{l}\text { Clinical } \\
\text { features }\end{array}$ & $\begin{array}{l}\text { Mycological } \\
\text { evidence } \\
\text { other than GM }\end{array}$ \\
\hline 1 & $\mathrm{x}$ & - & $\mathrm{x}$ \\
\hline 2 & $\mathrm{x}$ & - & $\mathrm{x}$ \\
\hline 3 & $\mathrm{x}$ & - & $\mathrm{x}$ \\
\hline 4 & $\mathrm{x}$ & - & $\mathrm{x}$ \\
\hline 5 & $\mathrm{x}$ & - & $\mathrm{x}$ \\
\hline 6 & $\mathrm{x}$ & - & $\mathrm{x}$ \\
\hline 7 & $\mathrm{x}$ & - & $\mathrm{x}$ \\
\hline 8 & $\mathrm{x}$ & - & $\mathrm{x}$ \\
\hline 9 & $\mathrm{x}$ & - & $\mathrm{x}$ \\
\hline 10 & $\mathrm{x}$ & - & $\mathrm{x}$ \\
\hline 11 & $\mathrm{x}$ & - & $\mathrm{x}$ \\
\hline 12 & - & $\mathrm{x}$ & $\mathrm{x}$ \\
\hline 13 & - & $\mathrm{x}$ & $\mathrm{x}$ \\
\hline
\end{tabular}

Note: no number of patient, IA invasive aspergillosis, grey Nortase group, yellow no Nortase group

\section{Comparison of GM levels and kinetic patterns}

In all the false-positive patients in group 1, GM antigenemia occurred during the administration of Nortase. Fourteen patients $(63.6 \%)$ already showed positive GM results in the first or second measurement after initiation of Nortase therapy. Five patients $(22.7 \%$ ) already showed negative GM results in the first or second measurement after cessation of Nortase therapy.

The median GM index in all true positive patients was 1.3, and in all false-positive patients it was 1.7. Statistical evaluation with the Mann-Whitney $U$ test showed no significant difference $(p=0.88)$ between these groups. There was also no significant difference in GM levels between true positive and false positive between each group (group $1(p=0.45)$, group $2(p=0.40))$.

\section{Antifungal therapy}

Systemic antifungal therapy was administered to 24 patients (80\%) in group 1, and to 31 patients $(88.6 \%)$ in group 2. They received voriconazole, liposomal amphotericin B, caspofungin, or isavuconazole depending on patient factors. According to our data, 16 (53.3\%) patients allocated to group 1 and two patients (5.7\%) allocated to group 2 retrospectively received antifungal therapy due to false-positive GM results. In contrast, none of the patients with evidence for IA was deprived of antifungal therapy in the two groups.

\section{Determination of GM and BDG levels in in vitro Nortase samples}

In vitro analysis of Nortase samples of three different batches suspended in $10 \mathrm{~mL}$ normal saline each yielded a median GM index of 2.32 (range: 2.25-7.01). The specimens were subjected to further analysis for fungal antigens by testing for the panfungal marker BDG. All Nortase batches were found to contain high concentrations of BDG (median $1034.8 \mathrm{pg} / \mathrm{mL}$ (range: 975-1034.8)).

\section{Discussion}

GM screening is a valuable tool for the early detection of IA [3, 4]. In immunocompromised patients, the test performance is known to be relatively good [7-9]. Our finding of a PPV of 0.83 for patients without Nortase therapy is in good agreement with the previously published data. However, we observed that $75 \%$ of our ICU patients receiving Nortase developed GM antigenemia. Deterioration of the test's performance by the administration of digestive enzymes has not been described before. This retrospective analysis aimed to determine whether the antigen-based diagnosis was indeed correct.

The probability of IA diagnosis was graded by applying the scientific criteria for invasive mold infections defined by the EORTC/MSG consensus group [2].GM seropositivity is a major factor (criterion "mycological evidence") for grading, but with respect to the question addressed by this study, we had to exclude antigen testing from the definitions, making them even more restrictive (modified categories: 
probable* and possible* IA; see Table 1). Notably, the EORTC/MSG criteria were developed for severely immunocompromised patients with cancer and for hematopoietic stem cell transplant recipients. These patients differ from typical ICU patients regarding disease presentation as well as test performances. The consensus group addressed this issue but was unable to generate recommendations for this special population $[2,11]$. Alternative algorithms have been proposed for ICU patients but are not yet used in clinical routine [12]. In our study, several patients met two of the three criteria for probable IA, but not the third criterion, i.e., a clinical feature. These patients were declared as presumable IA as their specific probability for IA was high. The reason for a lack of clinical features in these patients is obvious as differences in disease presentation between neutropenic cancer patients and ICU patients are well known by now. For instance, radiological findings in ventilated patients are nonspecific so broadening the radiological criteria seems valuable $[12,13]$. Therefore adding the category "presumable IA" allowed us to make the grading of ICU patients very close to the canonical EORTC/MSG guidelines (Table 1).

It is important to point out, though, that most of the IA patients (64.9\%) in our study were classified along the common EORTC/MSG categories proven, probable, and possible IA (still excluding GM antigenemia).

Our data suggest that a dramatically high proportion of 73.3\% of all patients receiving Nortase showed false-positive results in Aspergillus antigen testing (control group: 17.1\%).

However, how can we tell that they were false positive?

All GM positive patients received an intense diagnostic workup, which included microbiological testing such as cultures from different body sites, CT imaging, and histopathology of the lung. Autopsy was performed in two patients, with neither histology nor culture yielding any evidence for IA. Upon exclusion of GM positivity from the IA defining criteria, these 22 and 6 patients (Nortase vs. control group) did not even meet the criteria for possible*/presumable IA; they had no evidence for infection.

GM antigenemia kinetics underlined suspected false positivity as GM levels abruptly increased within a few days after the administration of Nortase and were resolved in many cases after its discontinuation.

In vitro testing of Nortase samples of different batches yielded highly positive results in the GM and BDG assays. In a critical care setting, DEFO are usually administered through an enteral feeding tube. Presumably, impaired mucosal integrity allows for GM translocation into the bloodstream resulting in seropositivity [14]. Intestinal hyperpermeability with translocation from the gut can often be seen in critical illness [15], possibly even more relevant in critical ill patients with cystic fibrosis [16]. This might explain why the influence of DEFO on the performance of the GM test has not been described before-it might not be a problem beyond ICU settings.

In our study, more than $80 \%$ of all patients who developed GM antigenemia received systemic antifungal therapy prior to the results of a further diagnostic workup. This is concordant with a preemptive strategy, meaning antifungal therapy based on biomarker surveillance [17]. According to our data, 16 patients allocated to group 1 (53.3\%), but only 2 patients allocated to group $2(5.7 \%)$, may have retrospectively received antifungal therapy due to possibly falsepositive GM results.

Our routine has been changed by these findings. The application of Nortase in patients who are at risk of IA must be strictly limited. Patients who develop GM antigenemia following DEFO application do not get antifungal therapy as long as there is no other sign of IA, as false positivity is probable. If there is any doubt, DEFO therapy should be discontinued and enteral nutrition must be initiated by peptide diets whose resorption is independent of pancreatic enzymes.

False positive GM antigenemia has been described before and was associated with the administration of generic piperacillin/tazobactam [18-22], hypercaloric drinks [23], antifungal prophylaxis [24, 25], and even ice-pop ingestion and pasta. In most cases, the proposed mechanism for false positivity was the contamination of the product with GM. Based on the literature and our in vitro results of GM and BDG testing, we assume that the applied purification techniques for digestive enzymes from fungal cultures cannot prevent contamination of the drug with cell wall polysaccharides. As in vitro testing of Nortase for BDG also turned out to be positive, this alternative antigen test cannot be expected to help confirm the diagnosis. Since BDG is a panfungal biomarker, which is also used for the diagnosis of Candida and Pneumocystis infections, we speculate that the administration of DEFO could also result in misdiagnosis of other fungal infections.

This study has certain limitations. First, the in vivo analysis has a retrospective study setting, based on a relatively low number of patients. Nevertheless, compared to previous studies that investigated potential sources of false-positive $\mathrm{GM}$, this number is rather high $[24,25]$. Second, the two groups differ greatly regarding age. This is linked to different underlying diseases, as DEFO are typically given to patients with end-stage cystic fibrosis following lung transplantation, who are most of the time of young age when receiving the new lung. An age- and diagnosis- dependent influence on the rate of false-positive GM tests appears not to be likely, though. Third, the case definitions of IA have been modified, resulting, in a deviation from the scientific gold standard. Therefore, a careful interpretation of the results is required, and comparability with other studies is limited. 


\section{Conclusions}

In conclusion, the GM assay allows the detection of Aspergillus antigen with high sensitivity and specificity in the serum of patients with IA who did not have Nortase therapy. Factors resulting in false-positive GM testing must be known to avoid misdiagnosis, unnecessary treatment, and extraneous costs. GM antigenemia in patients receiving degestive enzymes of fungal origin like Nortase should be interpreted with caution due to the significantly higher rate of falsepositive test results in contrast to patients without Nortase therapy. In our opinion, the Summary of Product characteristics need to be adjusted to draw the attention of clinicians to these new findings.

Author contributions IS and CS designed the study. IS was responsible for the acquisition of demographic and clinical data. IS, KD, and CS interpreted the results and wrote the manuscript. KD and JW performed the in vitro analysis. UL, MI, and MZ participated in the study design and interpretation of results. All authors meet the key authorship requirements and agree to be accountable for all aspects of the work, ensuring that questions related to the accuracy or integrity of any part of the work are appropriately investigated and resolved.

Funding Open Access funding provided by Projekt DEAL. This research did not receive any specific grant from funding agencies in the public, commercial, or not-for-profit sectors.

Availability of data and materials All data generated during this study are included in this article.

\section{Compliance with ethical standards}

Conflict of interests Upon manuscript submission, all authors declare that they have no competing interests.

Ethical approval and consent to participate Approval was obtained from the ethical review committee of the Ludwig-Maximilians-Universität (registration number 20-269).

Open Access This article is licensed under a Creative Commons Attribution 4.0 International License, which permits use, sharing, adaptation, distribution and reproduction in any medium or format, as long as you give appropriate credit to the original author(s) and the source, provide a link to the Creative Commons licence, and indicate if changes were made. The images or other third party material in this article are included in the article's Creative Commons licence, unless indicated otherwise in a credit line to the material. If material is not included in the article's Creative Commons licence and your intended use is not permitted by statutory regulation or exceeds the permitted use, you will need to obtain permission directly from the copyright holder. To view a copy of this licence, visit http://creativecommons.org/licenses/by/4.0/.

\section{References}

1. Lin CY, et al. Comparison of Clinical Manifestation, Diagnosis, and Outcomes of Invasive Pulmonary Aspergillosis and Pulmonary Mucormycosis. Microorganisms. 2019;7:531.
2. Donnelly JP, et al. Revision and update of the consensus definitions of invasive fungal disease from the European organization for research and treatment of cancer and the mycoses study group education and research consortium. Clin Infect Dis. 2019. https ://doi.org/10.1093/cid/ciz1008.

3. Patterson TF, et al. Executive summary: practice guidelines for the diagnosis and management of aspergillosis: 2016 update by the infectious diseases Society of America. Clin Infect Dis. 2016;63:433-42.

4. Ullmann AJ, et al. Diagnosis and management of Aspergillus diseases: executive summary of the 2017 ESCMID-ECMMERS guideline. Clin Microbiol Infect. 2018;24:e1-e38.

5. Gao Y, Soubani A. Advances in the diagnosis and management of pulmonary aspergillosis. Adv Respir Med. 2019;87:231-43.

6. Mennink-Kersten MA, Donnelly JP, Verweij PE. Detection of circulating galactomannan for the diagnosis and management of invasive aspergillosis. Lancet Infect Dis. 2004;4:349-57.

7. Leeflang MM, et al. Galactomannan detection for invasive aspergillosis in immunocompromised patients. Cochrane Database Syst Rev. 2015;12:CD007394.

8. Ku NS, et al. Diagnostic value of the serum galactomannan assay for invasive aspergillosis: it is less useful in non-haematological patients. Scand J Infect Dis. 2012;44:600-4.

9. Teering S, et al. Prognostic value of serum galactomannan in mixed ICU patients: a retrospective observational study. Anaesthesiol Intensive Ther. 2014;46:145-54.

10. Schneider MU, et al. Pancreatic enzyme replacement therapy: comparative effects of conventional and enteric-coated microspheric pancreatin and acid-stable fungal enzyme preparations on steatorrhoea in chronic pancreatitis. Hepatogastroenterology. 1985;32:97-102.

11. Bassetti M, et al. Developing definitions for invasive fungal diseases in critically ill adult patients in intensive care units. Protocol of the FUNgal infections Definitions in ICU patients (FUNDICU) project. Mycoses. 2019;62:310-9.

12. Blot SI, et al. A clinical algorithm to diagnose invasive pulmonary aspergillosis in critically ill patients. Am J Respir Crit Care Med. 2012;186:56-64.

13. Park SY, et al. Clinical and radiological features of invasive pulmonary aspergillosis in transplant recipients and neutropenic patients. Transpl Infect Dis. 2010;12:309-15.

14. Klingensmith NJ, Coopersmith CM. The Gut as the Motor of Multiple Organ Dysfunction in Critical Illness. Crit Care Clin. 2016;32:203-12.

15. Otani S, Coopersmith CM. Gut integrity in critical illness. J Intensive Care. 2019;7:17.

16. Gelfond D, Borowitz D. Gastrointestinal complications of cystic fibrosis. Clin Gastroenterol Hepatol. 2013;11:333-42.

17. Segal BH, Walsh TJ. Current approaches to diagnosis and treatment of invasive aspergillosis. Am J Respir Crit Care Med. 2006;173:707-17.

18. Demiraslan $\mathrm{H}$, et al. Assessing the risk of false positive serum galactomannan among patients receiving piperacillin/tazobactam for febrile neutropenia. Med Mycol. 2017;55:535-40.

19. Ortiz-Brizuela E, et al. False-positive results in the galactomannan Platelia Aspergillus assay with generic piperacillin/ tazobactam. Rev Iberoam Micol. 2019;36:51-2.

20. Alhambra A, et al. False positive galactomannan results in adult hematological patients treated with piperacillin-tazobactam. Rev Iberoam Micol. 2007;24:106-12.

21. Aubry A, et al. Occurrence and kinetics of false-positive Aspergillus galactomannan test results following treatment with betalactam antibiotics in patients with hematological disorders. J Clin Microbiol. 2006;44:389-94. 
22. Sulahian A, Touratier S, Ribaud P. False positive test for Aspergillus antigenemia related to concomitant administration of piperacillin and tazobactam. N Engl J Med. 2003;349:2366-7.

23. Rachow $\mathrm{T}$, et al. Case report: false positive elevated serumgalactomannan levels after autologous hematopoietic stem cell transplantation caused by oral nutritional supplements. Clin Case Rep. 2016;4:505-8.
24. Vena A, et al. The misleading effect of serum galactomannan testing in high-risk haematology patients receiving prophylaxis with micafungin. Clin Microbiol Infect. 1000e;23:1000e1-e4.

25. Duarte RF, et al. Serum galactomannan-based early detection of invasive aspergillosis in hematology patients receiving effective antimold prophylaxis. Clin Infect Dis. 2014;59:1696-702. 